\title{
Beyond insulin: On the Road to a Cure for Type 1 Diabetes
}

\author{
Jenna Capyk \\ (University of British Columbia) \\ News Reporter (HSI 2011-2012)
}

Dependence on daily insulin injections often begins in early childhood for those with type 1 diabetes, an autoimmune disorder that destroys insulin-secreting $\beta$-cells in the pancreas. The hormone insulin plays an essential role in blood glucose regulation by triggering uptake of blood glucose by body tissues. Glucose is a crucial energy source within our bodies and its level in the bloodstream is maintained within a very narrow range by insulin and other hormones. Low blood glucose starves vital organs and can lead to coma and death. In contrast, chronically high blood glucose results in damage to fine blood vessels and nerve fibres.

Type 1 diabetics are dependant on externally supplied insulin and must measure their blood glucose levels, calculate the correct amount of insulin to balance their food intake and planned activity level, and inject these insulin doses several

\section{While exogenous insulin therapies are life- saving treatments for diabetes, they fail to prevent its crippling complications, which can restrict daily activities and dramatically decrease lifespan.}

times daily. This therapy results in unhealthy fluctuations in blood glucose. Insulin pumps can be used to supply insulin via a catheter, but even this improved treatment leaves patients dependant on drugs and equipment, and does not fully recapitulate natural insulin production.

While exogenous insulin therapies are life-saving treatments for diabetes, they fail to prevent its crippling complications, which can restrict daily activities and dramatically decrease lifespan. These complications include cardiovascular disease, neuropathy, blindness, kidney disease, and lower limb amputation. Consequently, type 1 diabetics in North America predecease their non-diabetic peers by over a decade, on average.

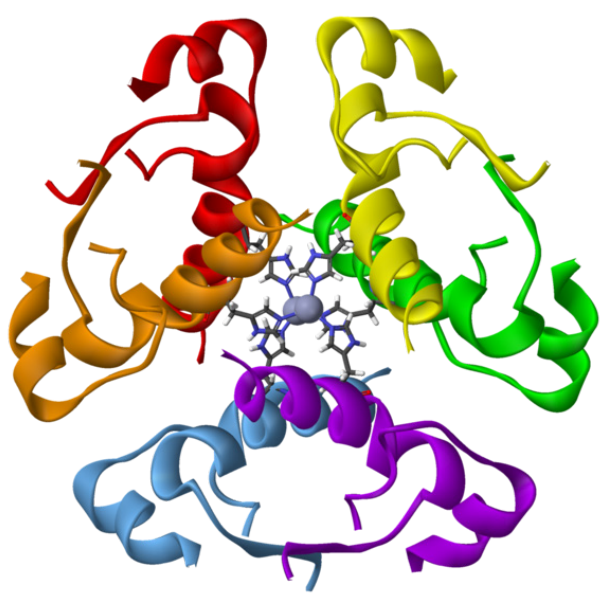

The hormone insulin plays an essential role in blood glucose regulation by triggering uptake of blood glucose by body tissues.

Alternatives treatments, which establish production and secretion of insulin within the patient's own body, hold great promise for the future of diabetes treatment. To learn more about the new treatments being developed by Canadian scientists, I talked to Dr. Timothy Kieffer, a principal investigator in the Diabetes Research Group in the Department of Cellular and Physiological Sciences at the University of British Columbia.

One area of research in Dr. Kieffer's laboratory involves using islet transplants to replace the $\beta$-cells destroyed in type 1 diabetes. The current islet transplant procedure, known as the "Edmonton protocol," entails purifying the $\beta$-cell-containing islets from the pancreas of a recently deceased donor and infusing these cells into the liver of a diabetic patient. The transplanted $\beta$-cells are able to sense glucose levels in their blood supply and release insulin, thus maintaining normal blood glucose levels.

Islet transplant surgery has great potential as a diabetes therapy, but it also has significant limitations. One major consideration, Dr. Kieffer points out, is the need for continuous immunosuppression to prevent rejection of the donor cells and to inhibit the type 1 diabetic autoimmune response against $\beta$-cells. Chronic immunosuppression carries significant risks. This surgery is therefore only recommended for diabetics with very poor blood glucose control, and is not a viable option for small children. Transplanted $\beta$-cells also lose function over time, with most 
patients relying once again upon injected insulin five years post-transplant. Additionally, severely limited supplies of both donor pancreases and facilities with the equipment and expertise needed to process them limit widespread practice of islet transplantation.

Dr. Kieffer's laboratory is one group working to produce $\beta$-cells from stem cells, eliminating the requirement for donor pancreases. He explains that diabetes is in a unique position among diseases under consideration for stem cell-based therapies: "There's limited evidence, for example, that with spinal cord injury you can transplant cells and reverse the disease. With diabetes... we know it works based on islet transplant." While such a renewable source of $\beta$-cells for transplant would be life-changing for many people, there is currently no established protocol for making stem cell-derived $\beta$ cells that exhibit a natural insulin secretion profile. According to Dr. Kieffer: "I do believe we're getting closer and that it will work, but we're not there yet."

Dr. Kieffer's group is also working on engineering other cells in the body to take over insulin production. Mammals have specialized intestinal cells that produce hormones secreted in response to ingested glucose. Release of these substances in the gut stimulates insulin secretion in the pancreas, and the blood levels of these hormones parallel that of insulin. Dr. Kieffer explains that, "If we can fool these gut cells to produce insulin, maybe we can reestablish the automatic meal-dependent pattern of insulin and therefore eliminate the need for insulin injections or $\beta$-cell transplant." It is an attractive concept with the potential to evade the anti- $\beta$-cell autoimmune response that characterizes type 1 diabetes. The Kieffer lab, in conjunction with the company enGene, is currently working toward producing a "gene pill" that can safely deliver the insulin gene into these gut cells and establish glucose-dependant insulin secretion. While several hurdles remain, including optimizing a safe gene delivery system and providing proof that there is no autoimmune response to the new insulinproducing cells, this approach has the potential for long- term efficacy with no dependence on exogenous insulin or immunosuppressive agents.

\section{Alternatives treatments, which establish} production and secretion of insulin within the patient's own body, hold great promise for the future of diabetes treatment.

Most patients with type 1 diabetes today face years of exogenous insulin therapy, but improvements in islet transplant technology and the prospect of gene-based therapies represent two distinct paths towards curative treatments for this devastating disease. Scientists are working toward reducing post-transplant dependence on immunosuppressive drugs by combining $\beta$-cell transplant with a more elegant form of immunosuppression. As for the limited supply of transplantable islet cells, Dr. Kieffer remarks, "looking into my crystal ball, I believe that in the not too-distant future there will be clinical trials of not donor-derived islets, but stem cell-derived islet cells going into patients." As he and his colleagues continue their work toward these goals, Dr. Kieffer expresses optimism for their eventual success: "I think that the parts are in place, and it's now just a matter of time."

\section{Acknowledgements}

Special Thanks to Dr. Tim Kieffer for his contribution to the above article.

\section{References}

1. Shapiro AM, Lakey JR, Ryan EA, Korbutt GS, Toth E, Warnock GL, Kneteman NM, Rajotte RV. New England Journal of Medicine. 2000. 343 (4): 230-238.

2. Kieffer TJ and Habener JF. Endocrine Reviews. 199920 (6): 876-913.

3. Weir GC, Cavelti-Weder C, Bonner-Weir S. Genome Medicine 3. 2011. (61).

4. Fujita Y, Cheung AT, Kieffer TJ. Pediatric Diabetes. 2004. 5:57-69.

5. Tuduri E and Kieffer TJ. Diabetes, Obesity and Metabolism. 2011. 13: 53-59.

6. World Health Organization Fact Sheet on Diabetes, August 2011, Sheet No 312.

7. Schaepelynck P, Darmon P, Molines L, Jannot-Lamotte MF, Treglia C, Raccah D. (2011) Diabetes Metab. 2011. Dec;37 Suppl 4:S85-93.

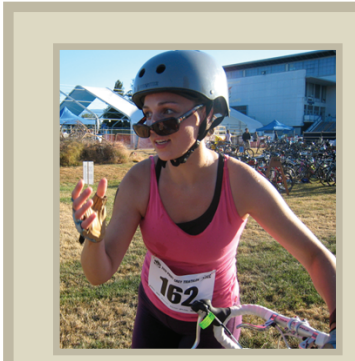

\section{Jenna Capyk}

Jenna Capyk is a recent PhD graduate from the University of British Columbia in Vancouver. Her graduate studies focused on the biochemistry of cholesterol degradation in the human pathogen Mycobacterium tuberculosis. She is a frequent podcast guest host where she enjoys making biology both accessible and entertaining. Jenna continues to engage in scientific communication through her position as a scientific marketing specialist at STEMCELL Technologies Inc., a Vancouver-based biotechnology company. 